\title{
Nonthermal Antiferromagnetic Order and Nonequilibrium Criticality in the Hubbard Model
}

\author{
Naoto Tsuji, ${ }^{1}$ Martin Eckstein, ${ }^{2}$ and Philipp Werner ${ }^{1}$ \\ ${ }^{1}$ Department of Physics, University of Fribourg, 1700 Fribourg, Switzerland \\ ${ }^{2}$ Max Planck Research Department for Structural Dynamics, University of Hamburg-CFEL, 22607 Hamburg, Germany
}

(Received 29 September 2012; revised manuscript received 25 January 2013; published 28 March 2013)

\begin{abstract}
We study dynamical phase transitions from antiferromagnetic to paramagnetic states driven by an interaction quench in the fermionic Hubbard model using the nonequilibrium dynamical mean-field theory. We identify two dynamical transition points where the relaxation behavior qualitatively changes: one corresponds to the thermal phase transition at which the order parameter decays critically slowly in a power law $\propto t^{-1 / 2}$, and the other is connected to the existence of nonthermal antiferromagnetic order in systems with effective temperature above the thermal critical temperature. The frequency of the amplitude mode extrapolates to zero as one approaches the nonthermal (quasi)critical point, and thermalization is significantly delayed by the trapping in the nonthermal state. A slow relaxation of the nonthermal order is followed by a faster thermalization process.
\end{abstract}

DOI: 10.1103/PhysRevLett.110.136404

PACS numbers: $71.10 . \mathrm{Fd}, 64.60 . \mathrm{Ht}$

In many physical systems out of equilibrium, phase transitions occur as a real-time process of symmetry breaking or symmetry recovery. Examples for such "dynamical phase transitions" include the evolution of the Universe [1], liquid helium [2], and photoinduced phase transition in solids [3-5]. The macroscopic aspects are often described by the time-dependent Ginzburg-Landau theory, where the order parameter is supposed to vary sufficiently slowly in time and space, so that the system can be considered to be locally close to thermal equilibrium. On the other hand, recent experimental developments of time-resolved measurement techniques in solids [6] and cold atoms [7] allow one to study dynamical phase transitions very far from equilibrium on the microscopic time scale of correlated quantum systems. In these cases, a "near-equilibrium" description might not be applicable. For instance, it has been recently suggested that superconductivity can be induced above the equilibrium critical temperature $\left(T_{c}\right)$ by coherently exciting certain lattice vibrations, and that it lasts for a relatively long time (a few tens of ps) before thermalization occurs [5]. This observation is reminiscent of the prethermalization phenomenon [8-11], or the dynamics in the presence of a nonthermal fixed point in relativistic quantum field theories [12]. A fundamental question that we pose here is if the existence of such a nonthermal fixed point in correlated condensed matter systems allows symmetry-broken states to survive above $T_{c}$, and how it affects the dynamics.

An important and still unresolved issue is how to characterize a nonequilibrium phase transition and its critical behavior for quantum systems [13,14]. Previous studies have in particular focused on the dynamics near quantum phase transitions in low dimensional systems (e.g., Refs. [15-18]). Higher dimensional systems are usually expected to show a thermal criticality out of equilibrium since quantum fluctuations are well suppressed. In this Letter, we study a dynamical phase transition for a simple microscopic model of correlated materials, namely the Hubbard model. In equilibrium, the model exhibits a phase transition from paramagnetic (PM) to antiferromagnetic (AFM) order [see the phase diagram in Fig. 1(a)]. By changing the interaction in time, we cross the phase boundary dynamically. In particular, we explore the weak-coupling regime of the Hubbard model (for the strong-coupling side, see our complementary work [19]). Contrary to the naive expectation, we find that the nonequilibrium relaxation behavior can be very different from the thermal one even in the large-dimensional limit. A new phenomenon that we demonstrate here is that in addition to the thermal critical point there exists one more quasicritical point (or sharp crossover) at which some time (energy) scale almost diverges (vanishes). Between these points, the system is trapped in a nonthermal "ordered" state [Fig. 1(b)], where the order parameter stays nonzero even though the effective temperature (which will be defined below) is above $T_{c}$.

The model Hamiltonian is given by

$$
H(t)=\sum_{\boldsymbol{k} \sigma} \epsilon_{\boldsymbol{k}} c_{\boldsymbol{k} \sigma}^{\dagger} c_{\boldsymbol{k} \sigma}+U(t) \sum_{i}\left(\hat{n}_{i \uparrow}-\frac{1}{2}\right)\left(\hat{n}_{i \downarrow}-\frac{1}{2}\right),
$$

where $\epsilon_{\boldsymbol{k}}$ is the band dispersion, $c_{\boldsymbol{k} \sigma}^{\dagger}\left(c_{\boldsymbol{k} \sigma}\right)$ is a creation (annihilation) operator of fermions with spin $\sigma, U$ is the (time-dependent) interaction strength, and $\hat{n}_{i \sigma}=c_{i \sigma}^{\dagger} c_{i \sigma}$. For convenience, we take a semicircular density of states, $D(\epsilon)=\sqrt{4-\left(\epsilon / t^{*}\right)^{2}} /\left(2 \pi t^{*}\right)$, and use $t^{*}\left(t^{*-1}\right)$ as the unit of energy (time). We only show results for the half-filling case. The initial state is in thermal equilibrium with temperature $T$, which is chosen such that the initial value of the staggered magnetization $m=\left\langle\left|\hat{n}_{\uparrow}-\hat{n}_{\mid}\right|\right\rangle$is 0.4 . The interaction is changed as $U(t)=U_{i}+\left(U_{f}-U_{i}\right) t / t_{q}(0 \leq t \leq$ $t_{q}$ ) with quench time $t_{q}=8$ fixed. The interaction quench can be implemented in cold atom systems with the use of 

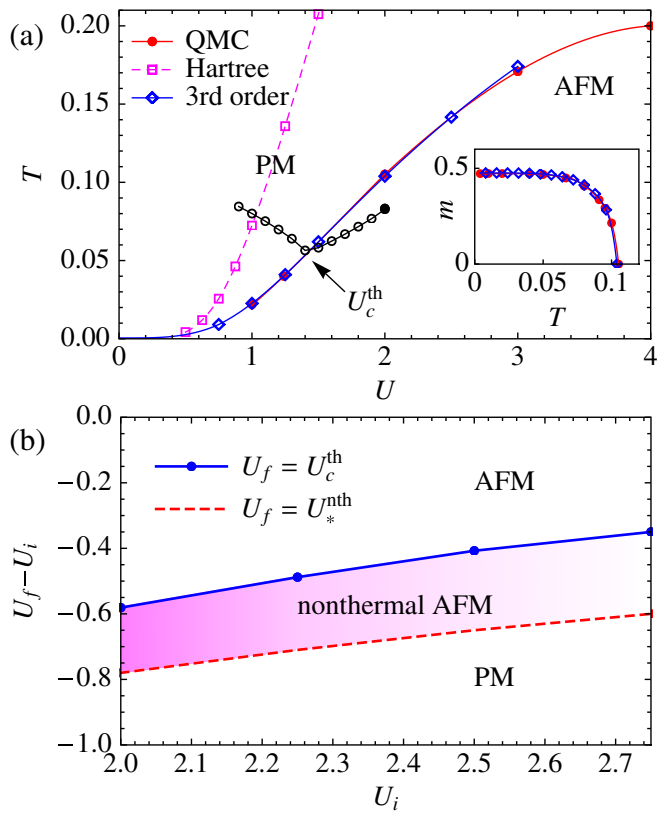

FIG. 1 (color online). (a) Equilibrium phase diagram of the Hubbard model in the weak-coupling regime at half filling, calculated by DMFT with several different impurity solvers. QMC data are taken from Ref. [25]. Effective temperatures for quenches from a fixed initial state $\left(U_{i}=2\right.$, black dot $)$ to various final states (open dots) are shown. Inset: Staggered magnetization $m$ as a function of $T$ at $U=2$. (b) Nonequilibrium phase diagram for a quench $U_{i} \rightarrow U_{f}$ with the fixed initial magnetization, $m(0)=0.4$. For $U_{f}>(<) U_{c}^{\text {th }}$, the system finally thermalizes to an AFM (PM) state. A nonthermal AFM order emerges in the colored region. The shading indicates the increasing lifetime of the nonthermal AFM state as $U_{i}$ is reduced.

the Feshbach resonance, or by modifying the depth of the lattice potential, and has also been proposed to be possible in solids driven by strong electric fields [20,21].

The time evolution of the Hubbard model with AFM order is studied with the nonequilibrium dynamical meanfield theory (DMFT) $[22,23]$. It becomes exact in the largedimensional limit [24], where the self-energy becomes local in space but keeps dynamical correlations. When one allows for AFM states in the single-site DMFT, the self-consistency condition reads $\Lambda_{\sigma}\left(t, t^{\prime}\right)=t^{* 2} G_{\bar{\sigma}}\left(t, t^{\prime}\right)$ $[19,22]\left[\Lambda_{\sigma}\left(t, t^{\prime}\right)\right.$ : hybridization function]. Since we are interested in the microscopic dynamics in a single magnetic domain, the system is assumed to take a spatially homogeneous configuration.

In order to treat the long-time behavior of symmetrybroken states, we adopt the third-order weak-coupling expansion as an impurity solver, i.e., expand all the self-energy diagrams, including the Hartree term, by Weiss Green functions $\mathcal{G}_{0 \sigma}\left(t, t^{\prime}\right)$ (bare propagators) up to third order in $U$. Although the bare expansion is not a conserving approximation in the sense of Baym and Kadanoff, it turns out to work remarkably well in the weak-coupling regime $(U \lesssim 3)$. For instance, the total energy is approximately
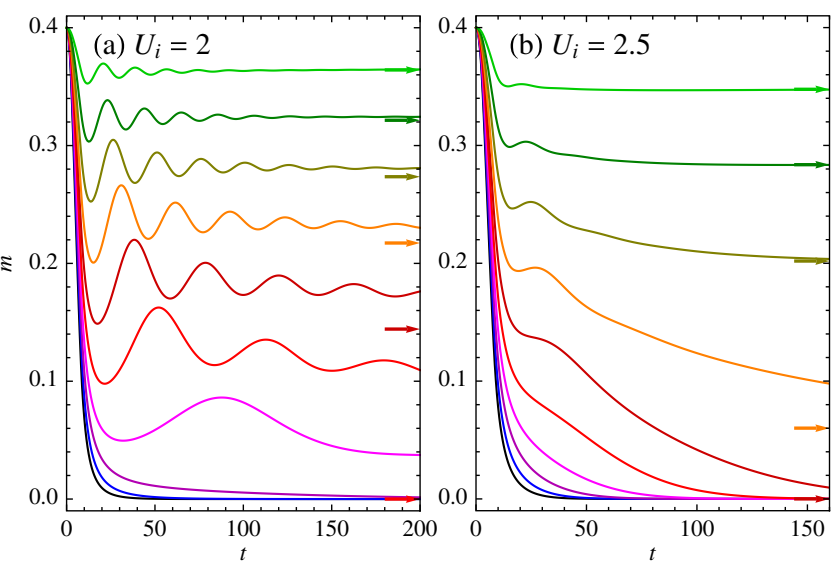

FIG. 2 (color online). Time evolution of $m$ for quenches (a) $U_{i}=2 \rightarrow U_{f}=1.0,1.1, \ldots, 1.9$ (from bottom to top), and (b) $U_{i}=2.5 \rightarrow U_{f}=1.5,1.6, \ldots, 2.4$. The arrows indicate the corresponding thermal values $m_{\text {th }}$ reached in the long-time limit.

conserved with negligibly small drifts. By comparison to quantum Monte Carlo (QMC) results [25], we confirmed that $T_{c}$ and $m$ in equilibrium are correctly reproduced [Fig. 1(a)], which is a considerable improvement from the Hartree approximation [Fig. 1(a)] and the second-order iterative perturbation theory [22].

Let us first look at results for quenches from $U_{i}=2$ to various $U_{f}\left(<U_{i}\right)$. As shown in Fig. 2(a), $m(t)$ quickly decreases after the quench due to the reduction of $U$, and starts to oscillate coherently (amplitude mode) with a slow drift. As $U_{f}$ decreases below $\sim 1.2$, the oscillation disappears, and $m$ exponentially decays to zero. Assuming that the nonintegrable Hubbard model thermalizes, the longtime limit of the order parameter is determined by the thermal value $m_{\mathrm{th}}$ at some effective temperature $T_{\text {eff }}$. Since the total energy is conserved after the quench $\left(t \geq t_{q}\right)$ in the isolated system, $T_{\text {eff }}$ is given by the temperature of the equilibrium system with the same total energy. The final thermalized states are plotted as open dots in Fig. 1(a). Since we are considering rather slow changes $\left(t_{q}=8\right)$ of $U$, the final states roughly keep track of the constant entropy curve [26].

The evaluated $m_{\text {th }}$ are indicated by arrows in Fig. 2, and are plotted as a function of $U_{f}$ in Fig. 4. One notices that the center of the oscillation of $m$ deviates more and more from $m_{\mathrm{th}}$ as $U_{f}$ is reduced. Surprisingly, at $U_{f}=U_{c}^{\mathrm{th}}=1.42$, where $m_{\text {th }}$ vanishes $\propto\left|U_{f}-U_{c}^{\text {th }}\right|^{\beta}$ (Fig. 4) with the meanfield exponent $\beta=\frac{1}{2}$ (thermal phase transition), $m$ still exhibits oscillations around a nonzero value for a long time. This suggests that the system is effectively trapped in a nonequilibrium quasisteady state, or close to a nonthermal fixed point, which allows for a long-lived symmetry-broken state with $T_{\text {eff }}$ above $T_{c}$. In the paramagnetic phase, the system shows prethermalization [9-11]; i.e., the momentum-integrated quantities such as the double occupancy thermalize faster than momentum-dependent 


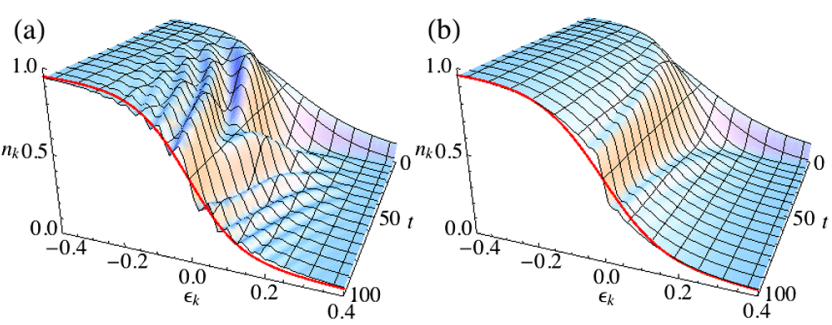

FIG. 3 (color online). Time evolution of the momentum distribution $n_{k}$ for quenches $U_{i}=2 \rightarrow$ (a) $U_{f}=1.4$ and (b) $U_{f}=1.2$. The curves at $t=100$ are thermal distributions achieved in the long-time limit.

quantities (e.g., the momentum distribution). Here a new observation is that the order parameter $m$, even though it is momentum integrated, also stays nonthermal, allowing the symmetry-broken state to survive for a long time. This can be attributed to the presence of "classical fluctuations" [12] in the Hartree term, which is absent in the paramagnetic phase.

To look at the qualitative change of the relaxation behavior around $U_{f} \sim 1.2$ more closely, we calculate the momentum distribution $n_{\boldsymbol{k}}(t) \equiv\left\langle c_{\boldsymbol{k} \sigma}^{\dagger}(t) c_{\boldsymbol{k} \sigma}(t)\right\rangle$ [27]. In Fig. 3, one can clearly see the qualitative difference of $n_{k}$ between (a) $U_{f}=1.4$ and (b) $U_{f}=1.2$. In the former case, waves are continuously generated at high energy, and cascade down to the lower energy region. They eventually reach the Fermi energy $\epsilon_{k}=0$, and lead to an oscillation of the slope $\partial_{\epsilon} n$ at $\epsilon_{k}=0$ [27]. In the latter case, the wave fronts never arrive at the Fermi energy but accumulate near $\epsilon_{k}=0$, which results in a steepening slope $\partial_{\epsilon} n$. This evolution is opposite to a heating effect, where an initially sharp momentum distribution is smeared out. Since the $n_{k}$ in Fig. 3(b) is very different from a thermal distribution [curve at $t=100$ in Fig. 3(b)] the fast relaxation of $m$ for $U_{f} \leq 1.2$ [Fig. 2(a)] is due to dephasing, not thermalization.

To characterize the nonthermal transition observed around $U \sim 1.2$ quantitatively, we evaluate the relaxation time $\tau_{\text {deph }}$ for the dephasing of $m(t)$ by fitting with $e^{-t / \tau_{\text {deph }}}$. As shown in Fig. 4(a), the dephasing critically slows down as $\tau_{\text {deph }} \propto\left|U_{f}-U_{*}^{\text {nth }}\right|^{-1}$ with $U_{*}^{\text {nth }}=1.23$ (nonthermal transition point). At $U_{f}=U_{*}^{\text {nth }}, m(t)$ shows a power-law decay of $t^{-1 / 2}$ until thermalization starts to take place around $t \sim 100$. This indicates that one more quasicritical point with an associated diverging time scale exists away from the thermal critical point $\left(U_{f}=U_{c}^{\mathrm{th}}\right)$. Moreover, a sharp kink is observed at $U_{f}=U_{*}^{\text {nth }}$ in the plot of the inverse of the steepest slope $\left(\partial_{\epsilon} n\right)^{-1}=\left(\max _{t}\left\{\left|\partial_{\epsilon} n(t)\right|\right\}\right)^{-1}$ at $\epsilon_{k}=0$ [Fig. 4(a)]. Because a true discontinuity in the momentum distribution function, with $\left(\partial_{\epsilon} n\right)^{-1}=0$, would correspond to a power-law decay of the density correlations in space, one may thus note that at the nonthermal critical point the system evolves through an almost
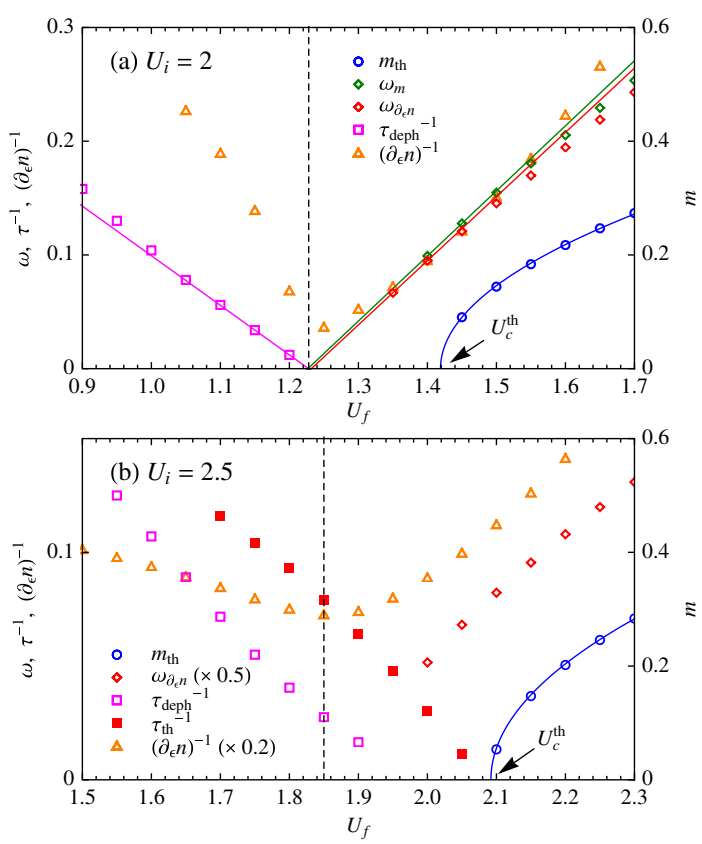

FIG. 4 (color online). Various quantities used to characterize the qualitative change of the behavior around $U_{f}=U_{c}^{\text {th }}$ and $U_{f}=U_{*}^{\text {nth }}$ (dashed lines) for quenches $U_{i} \rightarrow U_{f}$. Solid lines are guides for the eye.

"critical state" before thermalization sets in. We also determined the frequency $\omega_{m}$ of the amplitude mode of $m$ and the frequency $\omega_{\partial_{\epsilon} n}$ of the oscillation of $\partial_{\epsilon} n$ at $\epsilon_{k}=0$ for $U_{f}>U_{*}^{\text {nth }}$ by measuring the peak-to-dip distance of the oscillations. Note that near the critical point the period of the oscillation exceeds the lifetime $(\sim 100)$ of the trapped state, so that a meaningful measurement is not possible. However, the results in Fig. 4(a) indicate that $\omega_{m}$ and $\omega_{\partial_{\epsilon} n}$ extrapolate to zero as $\sim\left|U_{f}-U_{*}^{\text {nth }}\right|$. Based on this fact, we conclude that the amplitude mode is associated with the nonthermal fixed point, not with the thermal phase transition. This is not expected in the GinzburgLandau picture, where the oscillation disappears when the curvature of the free energy potential at the origin changes sign at the thermal critical point.

This quasicritical point (or sharp crossover) becomes an exact critical point in the weak-correlation limit, where the dynamics is described by the Hartree approximation. As we show in the Supplemental Material [27], the Hartree equation is mathematically equivalent to the timedependent BCS equation, which is known to be integrable with infinitely many conserved quantities $[28,29]$. There is a strict transition for the motion of the order parameter from damped oscillation to overdamped decay that is both associated with a diverging dephasing time (overdamped decay) and a vanishing of the amplitude-mode frequency. What we found here is that the qualitative aspects of the transition are maintained even in the regime where the Hartree approximation breaks down $(U \geqslant 0.5)$ due to 
quantum corrections from higher-order diagrams. In fact, the Hartree equation gives quantitatively very different results in this regime [27].

As one increases $U_{i}$, the system spends less time near the nonthermal fixed point, and thermalization occurs earlier. For $U_{i}=2.5$ [Fig. 2(b)], coherent amplitude oscillations are not visible anymore, and only a bump structure remains on a short time scale $(t \lesssim 30)$ for $U_{f}>1$.8. In this interaction regime the system does not show a clear signature of a transition, but a nonthermal crossover behavior is still seen in various quantities [Fig. 4(b)] around $U_{f}=U_{*}^{\text {nth }} \sim$ 1.85, which is estimated from the maximum of $\partial_{\epsilon} n$. For $U_{f}<U_{*}^{\text {nth }}$, we find that the order parameter $m$ shows a two-step relaxation [Fig. 5(a)]; i.e., the short-time and long-time dynamics have different exponential decay rates. The former is identified to be $\tau_{\text {deph }}$, since it is smoothly connected to what we have defined as $\tau_{\text {deph }}$ in the previous $U_{i}=2$ case. The latter is related to the thermal phase transition where $m_{\text {th }}$ disappears, hence denoted by $\tau_{\text {th }}$ [30]. The obtained $\tau_{\text {deph }}$ and $\tau_{\text {th }}$ are shown in Fig. 4(b). Interestingly, in most cases $\tau_{\text {deph }}$ is larger than $\tau_{\text {th }}$, that is, the slow dephasing of $m$ is followed by faster thermalization. Furthermore, thermalization is significantly delayed compared to $\tau_{\text {th }}$. At $U_{f}=1.9$, for example, $\tau_{\text {th }}=15.3$ while the delay time of thermalization is $>100$. This allows the order parameter to survive longer than the thermalization time constant.

Finally, let us examine the relaxation around the thermal critical point. Thermalization critically slows down as one approaches the thermal critical point [Fig. 4(b)] with

$$
\tau_{\mathrm{th}} \propto\left|U_{f}-U_{c}^{\mathrm{th}}\right|^{-1},
$$

which, unlike $\tau_{\text {deph }}$, remains even when the interaction is increased. Since the critical behavior around the thermal transition is universal, i.e., does not depend on details of the initial state or the the ramp protocol, it can be described by equilibrium properties. In fact, near a thermal (or quantum) critical point the relaxation time is known to behave as $\tau_{\text {th }} \sim\left|U_{f}-U_{c}^{\text {th }}\right|^{-z \nu}$ [13]. Here $\nu$ is the critical exponent that characterizes the divergence of the correlation length, $\xi \sim\left|U_{f}-U_{c}^{\mathrm{th}}\right|^{-\nu}$, and $z$ is the dynamical critical exponent. Our result (1) is consistent with the mean-field exponents $\nu=\frac{1}{2}$ and $z=2$ for nonconserved order parameters [13]. Exactly at the thermal critical point $\left(U_{f}=U_{c}^{\mathrm{th}}\right)$, the correlation time diverges, and the order parameter thermalizes in a power law. In Fig. 5(b), we show the log-log plot of $m$ around the thermal critical point $\left(U_{c}^{\text {th }}=2.40\right)$. The curve agrees very well with

$$
m \propto t^{-1 / 2}
$$

This is consistent to the prediction of the dynamical scaling ansatz [13], $m \sim t^{-\beta / z \nu}$, with the mean-field exponent $\beta=\frac{1}{2}$.

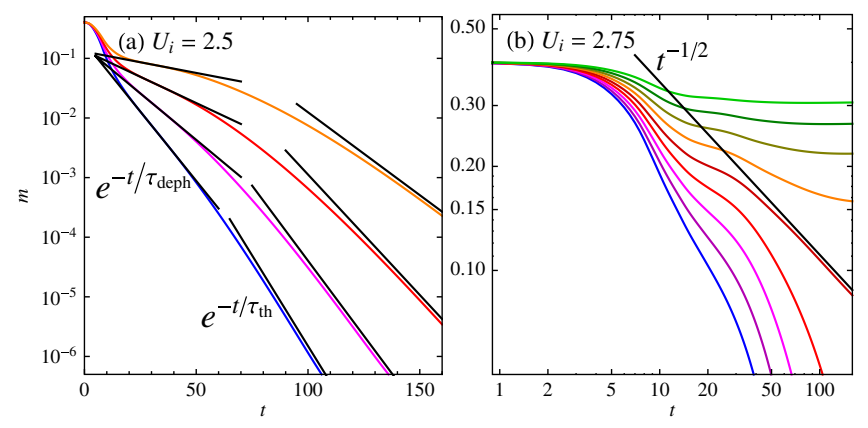

FIG. 5 (color online). (a) The log plot of $m$ for quenches $U_{i}=$ $2.5 \rightarrow U_{f}=1.6,1.7,1.8,1.9$ from bottom to top. The straight lines show fits of the two exponential relaxations. (b) The log-log plot of $m$ for quenches $U_{i}=2.75 \rightarrow U_{f}=2.2,2.25, \ldots, 2.6$ from bottom to top. The straight line shows the slope of a power-law decay $\propto t^{-1 / 2}$.

We summarize our results in a nonequilibrium phase diagram in Fig. 1(b). The results do not qualitatively change away from half filling [27] or with different initial $m$ or $T$. In fact, we numerically confirmed with the Hartree equation and the nonequilibrium DMFT that the slightly doped $(\$ 5 \%)$ system can be trapped in a nonthermal ordered state, and that the "critical" behavior at the nonthermal fixed point is the same. Our findings are applicable not only to antiferromagnetic order but also to superconductivity and charge density wave order if one translates the repulsive model to an attractive model [31]. An open question of practical importance is how to access this nonthermal fixed point. While we focused here on interaction quenches, the phenomenon is not specific to the particular quench protocol. For example, we have confirmed that a back-and-forth quench [27] gives similar nonthermal critical behavior with elevated $T_{\text {eff }}$, implying that the overall change of the interaction parameter is not essential. This universality nature of the phenomenon will open up a possible route to experimentally reach the nonthermal fixed point such as heating the system with laser irradiation. Since the order parameter is connected to the energy gap, the nonthermal order can be monitored with timeresolved optical and photoemission spectroscopies.

We thank H. Aoki, P. Barmettler, J. Berges, and T. Oka for fruitful discussions. The calculations were carried out on the Brutus cluster at ETH Zurich and on the UniFr cluster. We acknowledge support from the Swiss National Science Foundation (Grant No. PP0022-118866) and FP7/ERC Starting Grant No. 278023.

[1] T. W. B. Kibble, J. Phys. A 9, 1387 (1976).

[2] W. H. Zurek, Nature (London) 317, 505 (1985).

[3] F. Schmitt, P. S. Kirchmann, U. Bovensiepen, R. G. Moore, L. Rettig, M. Krenz, J.-H. Chu, N. Ru, L. Perfetti, D. H. Lu et al., Science 321, 1649 (2008). 
[4] R. Yusupov, T. Mertelj, V. V. Kabanov, S. Brazovskii, P. Kusar, J.-H. Chu, I. R. Fisher, and D. Mihailovic, Nat. Phys. 6, 681 (2010).

[5] D. Fausti, R. I. Tobey, N. Dean, S. Kaiser, A. Dienst, M. C. Hoffmann, S. Pyon, T. Takayama, H. Takagi, and A. Cavalleri, Science 331, 189 (2011).

[6] A. L. Cavalieri, N. Muller, T. Uphues, V. S. Yakovlev, A. Baltuska, B. Horvath, B. Schmidt, L. Blumel, R. Holzwarth, S. Hendel et al., Nature (London) 449, 1029 (2007).

[7] I. Bloch, J. Dalibard, and W. Zwerger, Rev. Mod. Phys. 80, 885 (2008).

[8] J. Berges, S. Borsányi, and C. Wetterich, Phys. Rev. Lett. 93, 142002 (2004).

[9] M. Moeckel and S. Kehrein, Phys. Rev. Lett. 100, 175702 (2008).

[10] M. Eckstein, M. Kollar, and P. Werner, Phys. Rev. Lett. 103, 056403 (2009).

[11] M. Kollar, F. A. Wolf, and M. Eckstein, Phys. Rev. B 84, 054304 (2011).

[12] J. Berges, A. Rothkopf, and J. Schmidt, Phys. Rev. Lett. 101, 041603 (2008).

[13] P. C. Hohenberg and B. I. Halperin, Rev. Mod. Phys. 49, 435 (1977).

[14] A. Polkovnikov, K. Sengupta, A. Silva, and M. Vengalattore, Rev. Mod. Phys. 83, 863 (2011).

[15] D. E. Feldman, Phys. Rev. Lett. 95, 177201 (2005).

[16] A. Mitra, S. Takei, Y. B. Kim, and A. J. Millis, Phys. Rev. Lett. 97, 236808 (2006).

[17] C. Kollath, A. M. Läuchli, and E. Altman, Phys. Rev. Lett. 98, 180601 (2007).
[18] P. Barmettler, M. Punk, V. Gritsev, E. Demler, and E. Altman, Phys. Rev. Lett. 102, 130603 (2009).

[19] P. Werner, N. Tsuji, and M. Eckstein, Phys. Rev. B 86, 205101 (2012).

[20] N. Tsuji, T. Oka, P. Werner, and H. Aoki, Phys. Rev. Lett. 106, 236401 (2011).

[21] N. Tsuji, T. Oka, H. Aoki, and P. Werner, Phys. Rev. B 85, 155124 (2012).

[22] A. Georges, G. Kotliar, W. Krauth, and M. J. Rozenberg, Rev. Mod. Phys. 68, 13 (1996).

[23] J. K. Freericks, V. M. Turkowski, and V. Zlatić, Phys. Rev. Lett. 97, 266408 (2006).

[24] W. Metzner and D. Vollhardt, Phys. Rev. Lett. 62, 324 (1989).

[25] A. Koga and P. Werner, Phys. Rev. A 84, 023638 (2011).

[26] F. Werner, O. Parcollet, A. Georges, and S. R. Hassan, Phys. Rev. Lett. 95, 056401 (2005).

[27] See Supplemental Material at http://link.aps.org/ supplemental/10.1103/PhysRevLett.110.136404 for the Hartree approximation, the momentum distribution function, and the quench protocol dependence.

[28] R. A. Barankov and L. S. Levitov, Phys. Rev. Lett. 96, 230403 (2006).

[29] E. A. Yuzbashyan and M. Dzero, Phys. Rev. Lett. 96, 230404 (2006).

[30] In the previous case $\left(U_{i}=2\right)$, the crossover from dephasing to thermalization could not be observed, because it occurs at times which cannot be reached in our simulation.

[31] H. Shiba, Prog. Theor. Phys. 48, 2171 (1972). 